\title{
Servicio público de televisión y patrimonio audiovisual: el proyecto VideoActive
}

\author{
Por David Fernández-Quijada y Montse Fortino
}

\begin{abstract}
Resumen: La necesidad de conservar los archivos televisivos y las posibilidades de difusión derivadas de la digitalización han generado un creciente interés debido al despliegue de plataformas desde las que proporcionar acceso a estos contenidos. Una de estas plataformas es VideoActive, un proyecto colaborativo paneuropeo con una aproximación temática tanto a la historia de Europa a través de la televisión como a la historia de la televisión europea, que tiene entre sus metas establecer puentes de colaboración con el mundo académico. El consorcio ha puesto en marcha su propia infraestructura técnica para suministrar hasta 10.000 piezas de diez archivos televisivos europeos, entre ellos Televisió de Catalunya, el operador público catalán que de esta manera busca proyectar internacionalmente la cultura catalana.

Palabras clave: Servicio público de radiodifusión, Patrimonio audiovisual, Archivos audiovisuales, Archivos televisivos, VideoActive, Televisió de Catalunya.
\end{abstract}

Title: The VideoActive project: Television as public service and audiovisual patrimony

Abstract: The need to preserve audiovisual archives and the possibilities for their dissemination resulting from digitisation have generated a growing interest in the development of platforms to provide access to their content. One of these platforms is VideoActive, a pan-European collaborative project that has developed a thematic approach to European history through TV and to the history of European TV. One of its key objectives is to encourage collaboration with the academic world. The consortium has developed its own technical infrastructure to offer at least 10,000 items from 10 different European archives, including that of Televisió de Catalunya, the Catalan public service broadcaster that seeks to contribute to international awareness of Catalan culture.

Keywords: Public service broadcasting, Audiovisual heritage, Audiovisual archives, Television archives, VideoActive, Televisió de Catalunya.

Fernández-Quijada, David; Fortino, Montse. "Servicio público de televisión y patrimonio audiovisual: el proyecto VideoActive". El profesional de la información, 2009, septiembre-octubre, v. 18, n. 5, pp. 545-551.

DOI: 10.3145/epi.2009.sep.09

\section{Servicio público y patrimonio audiovisual}

MEDIO DE CONSUMO MASIVO por excelencia, la televisión es el referente cultural, estético e informativo contemporáneo de más amplia difusión.

Contribuye por tanto a nuestra concepción del mundo, presente y pasado. De ahí la importancia de preservar sus fondos como memoria cultural e histórica. Una tarea que, además, les corresponde a los operadores de televisión como parte de la función de servicio público que tienen adjudicada.

Esta idea no siempre se plasma en la realidad, pues los canales pri- vados en general tienen poca predisposición a hacerlo. En cambio algunos operadores televisivos públicos están construyendo un archivo que se configura como parte fundamental de la memoria histórica del país.

"El archivo de un radiodifusor refleja la evolución de sus contenidos a lo largo del tiempo y cumple con la función de repositorio como parte de la memoria común de una sociedad" 
Esto implica una serie de responsabilidades notables, desde su efectiva conservación con las máximas condiciones de seguridad hasta la posible consulta de ese archivo por investigadores especializados o por la ciudadanía en general.

La importancia de las televisiones públicas en este campo no radica solamente en el hecho de que posean por razones históricas los archivos audiovisuales más extensos, sino también en que la lógica utilitarista del libre mercado que siguen las televisiones privadas no garantiza una adecuada preservación del patrimonio audiovisual que, por otra parte, conlleva altos costes. Esta tarea que normalmente recae sobre los correspondientes departamentos de documentación, forma parte cada vez más de las funciones del servicio público que le son asignadas en ese contrato social que se establece entre medios públicos y ciudadanos.

Legalmente hay pocas obligaciones al respecto para los radiodifusores y nada cercano a la idea del depósito legal que rige por ejemplo para los libros. La principal novedad en los últimos años la constituye la Ley 17/2006, de 5 de junio, de la radio y la televisión de titularidad estatal, que regula al operador público Radio Televisión Española, y en la que se cita entre sus funciones de servicio público la de "velar por la conservación de los archivos históricos audiovisuales", lo que se traduce en el artículo 52 de su preceptivo Mandato-Marco, dedicado al patrimonio audiovisual y en el que, entre otras cosas, se establece que "la Corporación Rtve tiene la obligación, en el período de vigencia del presente Mandato-Marco, de digitalizar, conservar y preservar en condiciones óptimas para su uso y consulta, el archivo histórico heredado de Rtve" y también que "la Corporación Rtve facilitará que esos archivos tengan presencia en bus- cadores y puedan ser visualizados o escuchados a través de las redes telemáticas, fijas y móviles".

http://www.boe.es/boe/dias/2006/ 06/06/pdfs/A21207-21218.pdf

http://www.boe.es/boe/dias/2008/ 06/30/pdfs/A28833-28843.pdf

El archivo de un radiodifusor refleja la evolución de sus contenidos a lo largo del tiempo y, como tal, cumple con la relevante función de repositorio. La memoria colectiva proporciona a una sociedad la posibilidad de satisfacer su necesidad simbólica de establecer un lazo con la historia de la comunidad (Lloyd, 2007). Los archivos televisivos más antiguos se corresponden con materiales cinematográficos utilizados en el medio, tales como noticiarios rodados en soporte químico. No fue hasta la invención en 1956 de la cinta magnética de dos pulgadas por la casa Ampex que se dio la posibilidad de sistematizar un archivo, aunque la falta de conciencia de memoria histórica ha privado en gran medida a los historiadores de esta fuente (Gómez-Alonso, 2004). En otros casos, la dificultad de acceder físicamente a los archivos ha restringido su posible consulta $\mathrm{y}$ utilización.

Consultar los archivos televisivos ha resultado históricamente una tarea complicada. Sin embargo, el tránsito al entorno digital se presenta como "el momento de que los archivos audiovisuales, en especial los de las televisiones públicas, se replanteen su misión, sus objetivos y lleven a cabo estrategias de comunicación" (Agirreazaldegi-Berriozabal, 2007, p. 440). En el caso de internet "significa liderar el proceso de coordinación de las diversas instituciones, sobre todo las públicas, en la gestión de la memoria digital, fuente de la información del futuro" (De-Moragas; Prado, 2000, p. 392). Además, la accesibilidad en línea constituye hoy en día una condición indispensable para optimizar los beneficios que pueden extraer de estos contenidos los ciudadanos y los colectivos sociales. Así lo han entendido radiodifusores públicos como la $B B C$ británica con su servicio BBC Motion Gallery o la $R A I$ italiana, así como el gran centro documental francés, el Institut National de l'Audiovisuel (INA).

http://www.bbcmotiongallery.com http://www.rai.tv

http://www.ina.fr/archivespourtous/

Desde una perspectiva continental, la Comisión Europea ha venido trabajando en la conservación y expansión del patrimonio cultural europeo. A modo de ejemplo, la iniciativa $i 2010$ sobre bibliotecas digitales recuerda que "la presencia en línea de este material procedente de distintas culturas y expresado en diferentes lenguas permitirá a los ciudadanos apreciar más fácilmente su propio patrimonio cultural y el de los demás países europeos y utilizarlo con fines de estudio, trabajo u ocio. Contribuirá, de esta manera, a complementar y apoyar los objetivos de la actuación de la Unión Europea en el ámbito de la cultura" (Comisión de las Comunidades Europeas, 2005, p. 4). La multiculturalidad y el multilingüismo de las expresiones culturales afloran pues como elementos centrales de i2010.

\section{"VideoActive pretende mostrar las similitudes y las diferencias en la presentación televisiva de la realidad social y cultural europea"}

\section{EI proyecto VideoActive}

En este contexto de progresiva concienciación sobre la necesidad de preservar y facilitar la difusión del patrimonio audiovisual, la llegada de la digitalización ha impulsado el surgimiento de varios proyectos 
colaborativos. La colaboración entre diferentes organismos dedicados a la preservación de documentos ha sido una constante desde el siglo pasado, pero la aparición de las tecnologías digitales ha convertido en más aguda esta necesidad por las nuevas habilidades que requiere el tratamiento de estos materiales y las dificultades por asumirlas todas bajo un mismo techo (Besser, 2007). Esta voluntad de trabajo conjunto se reflejó por ejemplo en el proyecto Presto (Teruggi, 2004; Wright, 2004), centrado en el análisis de tecnologías específicas para la digitalización de archivos audiovisuales.

\section{http://www.prestospace.org}

En Presto participaban algunos radiodifusores que se volverían a encontrar en Birth (Building an Interactive Research and Delivery Network for Television Heritage), otro proyecto de alcance continental nacido del mismo espíritu colaborativo (Hecht; O'Dwyer; Oomen; Scharinger, 2004) y que construyó la base técnica sobre la que trabaja el proyecto VideoActive.

http://www.birth-of-tv.org http://www.videoactive.eu

VideoActive muestra las similitudes pero también las diferencias en la presentación televisiva de la realidad social y cultural europea, así como el rol histórico que ha jugado la televisión en la creación de esta experiencia común. Financiado con más de 2,5 millones de euros por el programa eContentPlus de la Comisión Europea, VideoActive comenzó el 1 de septiembre de 2006 para un período de 36 meses que finalizó el pasado 31 de agosto de 2009.

http://ec.europa.eu/information society/activities/econtentplus/ programme/index_en.htm

\subsection{Contenido}

Los documentos audiovisuales que se pueden consultar en Video-
Active pertenecen a los archivos de las emisoras públicas British Broadcasting Corporation (BBC), Deutsche Welle $(D W)$, Danmarks Radio (DR), Österreichischer Rundfunk $(O R F)$, Radio Télévision Belge de la Communauté Française (RTBF), Vlaamse Radio- en Televisieomroep (VRT) y Televisió de Catalunya (TVC). También aportan sus fondos otros entes como el Nederlands Instituut voor Beeld en Geluid, el húngaro Neumann János Digitális Könyvtár és Multimédia Központ Kht., el italiano Istituto Luce, el sueco Sveriges Nationalarkiv för Audiovisuella Medier, el griego Hellenic National Audiovisual Archive (Henaa), el francés Institut National de l'Audiovisuel (INA) y la compañía británica Moving Image Communications Ltd. Junto a estos proveedores de contenidos, el peso técnico del proyecto recae sobre la compañía holandesa Noterik $B V$, que ha diseñado el portal y gestiona el material audiovisual, y la National Technical University of Athens, que gestiona los metadatos y el tesauro. Además, las facultades de comunicación de la Universiteit Utrecht y del Royal Holloway de la University of London asesoran en cuanto a los contenidos.

La selección de contenidos ha sido un aspecto clave en el que se ha trabajado desde el primer momento dada la variedad de archivos participantes, así como las diferencias en materia de propiedad intelectual y de disponibilidad de metadatos. La aproximación a los contenidos ha sido doble: abrir una ventana a la historia de Europa en la televisión y, por otro lado, mostrar la historia de la televisión europea. Este último aspecto se complementa además con artículos y textos elaborados para ayudar a comprender la historia televisiva en el continente.

http://videoactive.files.wordpress. com/2007/10/23_content_selection_ strategy_report.pdf

\section{"VideoActive muestra la historia de Europa en la televisión, y la historia de la televisión europea"}

Los materiales van acompañados de metadatos que ayudan en una tarea comparativa a la que también contribuye la propia política de selección de contenidos: priman aquellos que conllevan una comparación entre las diferentes culturas europeas, por ejemplo, el proceso de integración continental, la guerra fría o las distintas festividades nacionales. Así, esta aproximación temática se centra en aquellos materiales televisivos que tienen mayor capacidad de dar a conocer y ayudar a comprender la historia común de los europeos y las expresiones culturales del continente. Cada uno de los diez archivos participantes se ha comprometido a aportar hasta 1.000 piezas, algunas simplemente gráficas pero en su mayor parte imágenes en movimiento, lo que totaliza 10.000 piezas, de duración variable. En todo caso, una ínfima parte de lo disponible en sus archivos. El período de tiempo que abarcan los contenidos se inicia en 1907 con una filmación del día nacional danés, y llega hasta nuestros días.

\subsection{Tecnología}

La plataforma tecnológica del proyecto consta de tres partes bien diferenciadas: la aplicación con la que los participantes aportan sus contenidos, los servidores en los que se aloja el material digitalizado y el portal, el front-end con el público.

La solución diseñada para el proceso de contribución de archivos es el primer elemento a tener en cuenta. Los archivos se han gestionado con independencia de los metadatos. Los primeros se enviaban a los servidores de destino. Para los metadatos, la mayoría de los pro- 
veedores de contenidos han optado por introducirlos directamente mediante una interfaz web implementada como back-office en el portal de VideoActive. Otros, como TVC o el Istituto Luce, han optado por preparar la información en una base de datos propia para después exportarla al portal mediante ficheros excel.

El segundo elemento técnico sobre el que se sostiene VideoActive son los servidores. Noterik mantiene un servidor central en Holanda, pero las diferencias en las regulaciones nacionales no han permitido a algunos archivos almacenar sus piezas en servidores ubicados en el extranjero, como es el caso del Istituto Luce, que gestiona su propio servidor. La falta de una regulación común sobre propiedad intelectual ha obligado a flexibilizar este aspecto $y$, en general, ha originado algunos de los principales contratiempos en el cumplimiento del calendario previsto. Sea desde una localización u otra, el material videográfico se codifica a $25 \mathrm{fps}$ en mpeg $4 / \mathrm{h} .264$ a una resolución de $640 \times 480$ píxeles, lo que asegura una buena calidad incluso si la reproducción se realiza a pantalla completa. El audio, por su parte, se codifica en mp3 a $96 \mathrm{~Kb} / \mathrm{s}$.

El portal constituye el tercer y último elemento de la plataforma tecnológica. La primera versión beta del mismo fue puesta en mar- cha en febrero de 2008 y lanzada públicamente el 16 de septiembre del mismo año. Dado su carácter abierto, la consulta a sus contenidos puede beneficiar a cualquier ciudadano. No obstante, su utilización en entornos educativos ha sido uno de los motores que ha impulsado el proyecto. No sólo en la educación universitaria sino también como material de trabajo para la educación preuniversitaria.

La interfaz de consulta web de VideoActive es similar a la de otros sistemas de búsqueda ampliamente difundidos en la Red. La búsqueda avanzada, además, pone a disposición del público el contenido en función de idioma,

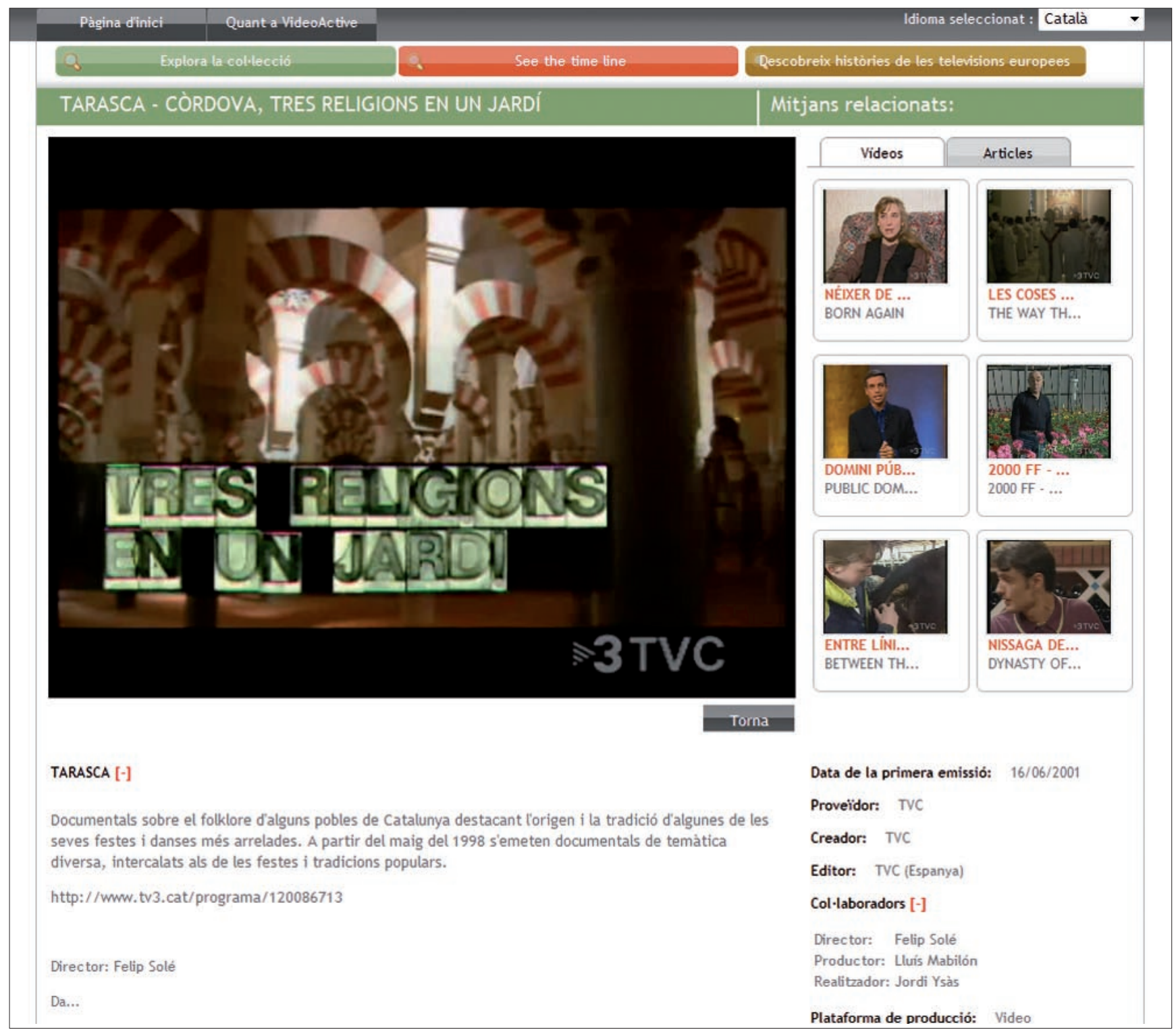

Figura 1. Interfaz web de VideoActive 
tema, archivo propietario del material, género televisivo, década de producción, fecha de emisión, etc. La navegación se puede efectuar hasta en diez idiomas diferentes: inglés, alemán, francés, italiano, holandés, griego, húngaro, danés, sueco y catalán. El propósito declarado de esta aproximación plurilinguiística es "reforzar la comprensión de las historias compartidas y las interrelaciones que han dado forma a la memoria y a la identidad europea colectiva, y a la vez conmemorar la dimensión multicultural que también ha forjado la ciudadanía europea".

http://www.videoactive.eu/Video Active/About.do?menu_page= menu-about

Para el tesauro se utiliza ThesauriX, que posibilita anotación multilingüe, de manera que los interesados pueden acceder a los contenidos con independencia del idioma en que éstos se encuentren. Los metadatos también se representan en rdf (resource description framework) y están disponibles públicamente mediante el protocolo $O A I-M P H$.

Más allá del propio portal de $V i$ deoActive, los contenidos son accesibles desde Europeana, la biblioteca digital paneuropea lanzada en 2008, en la que participa el consorcio que está detrás de VideoActive. Esta colaboración con una iniciativa que ha de convertirse en central en el entorno de internet contribuye a aumentar la visibilidad del material disponible en el portal.

\subsection{Vínculo con el mundo acadé- mico}

La selección del contenido se ha hecho en gran medida pensan- do en posibles aplicaciones para el mundo académico, como se ha dicho. Particularmente para el campo de los estudios de comunicación, por las facilidades que supone para los análisis comparativos textuales y estéticos, así como por el material que se aporta sobre la historia de la televisión en el continente europeo. No es casual que VideoActive colabore con la European Television History Network (ETHN), una red europea de académicos que investiga sobre la historia de la televisión y cuyos miembros han elaborado los textos del portal que contextualizan la historia televisiva europea. El material audiovisual disponible, además, puede resultar de gran utilidad para otros estudios desde la historia, las humanidades o la ciencia política (sobre el proceso de integración europea, por ejemplo).

La aproximación comparada a la historia televisiva no ha sido habitual en los estudios de comunicación, en gran parte por las diferencias idiomáticas y la dificultad para acceder a los materiales de base que harían posible este análisis comparativo. $\mathrm{Al}$ contar con VideoActive como fuente se abre una primera puerta a la elaboración de nuevas teorías y metodologías de aproximación a la televisión como objeto de estudio. Tal como se expone en la estrategia de selección del contenido del portal, "el tema del valor historiográfico [prioridades y necesidades a la hora de seleccionar los contenidos] es problemático y particularmente complejo ya que inevitablemente cambia en función de las fronteras nacionales, los sistemas radiotelevisivos y las disciplinas académicas".

\section{"Hay cooperación para la preservación del patrimonio audiovisual entre organizaciones de diferentes países, operadores y documentalistas e investigadores"}

http://videoactive.files.wordpress. com/2007/10/23_content_selection_ strategy_report.pdf

\section{Contribución de Televisió de Catalunya}

En este proyecto participa $T e$ levisió de Catalunya (TVC), el operador público autonómico catalán, que en los últimos años se ha distinguido por una activa política de incorporación e impulso de novedades tecnológicas para el cumplimiento de su función de servicio público (Prado; Fernández-Quijada, 2006).

\section{"En VideoActive, TVC pone al alcance del público parte de su fondo en un doble contexto: europeo y televisivo"}

El archivo de $T V C$ ha sido tradicionalmente de uso interno y se ha orientado desde sus orígenes a la preservación y reutilización del fondo en la propia producción. Siempre se ha mantenido abierto a la colaboración con investigadores $\mathrm{y}$ académicos y ha dispuesto del servicio de venta de copias físicas de programas. Desde 2004 funciona además un servicio de vídeo bajo demanda en internet, 3 a la carta, que ya proporciona una parte de sus contenidos de manera gratuita, una tarea facilitada por el proceso de digitalización del fondo documental llevado a cabo en los últimos años y por la integración de los procesos digitales en el programa de gestión de contenidos implementado en el centro, Digition Suite.

http://www.tv3.cat/seccio/ 3alacartal

La participación de TVC en $V i$ deoActive es una manera de poner al alcance del público parte de su fondo desde una doble perspecti- 
va: primero, en un contexto europeo, de manera que también sea una forma de proyectar la historia y la cultura catalanas al exterior y, en segundo lugar, en un contexto televisivo, gracias al trabajo específico que desde este operador se ha hecho para asignar metadatos a todos sus materiales. El trabajo se ha vehiculado mediante una documentalista encargada de seleccionar los ítems, añadir los metadatos y crear los clips de vídeo. Otras personas han colaborado en tareas auxiliares como las traducciones, el mantenimiento del tesauro o la transferencia de archivos.

Se han seleccionado piezas sobre las 34 materias fijadas, de forma más o menos equitativa entre las tres décadas de historia de TVC. La modernidad fue un factor diferenciador respecto al resto de los operadores del consorcio VideoActive, en su mayor parte antiguos monopolios fundadores de la televisión -y de la radio- en sus respectivos países.

En la selección también se ha buscado un equilibrio entre géneros televisivos, de manera que se pudieran encontrar piezas informativas pero también documentales, infantiles, ficción, spots publicitarios, etc. Además, al tratarse de un proyecto europeo se han primado los enfoques comparativos que proponían algunos programas históricos como Camí de l'euro o Estampes europees.

\section{Conclusiones}

En el proyecto VideoActive se manifiesta una triple voluntad de cooperación para la preservación del patrimonio audiovisual: primero, transnacional entre organizaciones de diferentes países; en segundo término, entre operadores y organismos públicos y empresas comerciales, y finalmente, interdisciplinar entre documentalistas e investigadores del campo de la comunicación.

Resulta también significativa la necesidad de crear un consorcio ad hoc para la puesta en marcha de un proyecto paneuropeo de esta singularidad, dada la existencia de una organización entre cuyas funciones cabría esperar precisamente la de generar estrategias para la conservación del patrimonio de sus asociados. Se trata de la Unión Europea de Radiodifusión (UER), formada básicamente por los operadores públicos europeos -y algunos privados- pero que no ha desplegado ningún proyecto similar a $\mathrm{Vi}$ deoActive, a pesar de que la mayoría de los miembros de éste último pertenecen también a la UER. No es el caso de TVC, que figura como único representante español en el proyecto a pesar de que el mayor archivo televisivo del país es el de Radio Televisión Española.

\begin{tabular}{|c|c|c|c|c|c|c|c|}
\hline \multirow{2}{*}{ Temas } & \multicolumn{3}{|c|}{ Décadas } & \multirow{2}{*}{ Temas } & \multicolumn{3}{|c|}{ Décadas } \\
\hline & 80 & 90 & 00 & & 80 & 90 & 00 \\
\hline Agricultura & 3 & 7 & 19 & Hogar & 11 & 12 & 16 \\
\hline Artes y cultura & 10 & 11 & 14 & Ocio & 4 & 10 & 6 \\
\hline Catástrofes & 4 & 8 & 15 & Vida urbana & 5 & 10 & 14 \\
\hline Guerra fría & 5 & 13 & 11 & Industria & 12 & 7 & 17 \\
\hline Sociedad de consumo & 5 & 7 & 25 & Fiestas nacionales & 7 & 9 & 4 \\
\hline $\begin{array}{l}\text { Programas de TV } \\
\text { controvertidos }\end{array}$ & 0 & 4 & 7 & Religión & 5 & 5 & 11 \\
\hline Crimen & 5 & 7 & 13 & Revolución sexual & 3 & 10 & 7 \\
\hline Democratización & 4 & 6 & 26 & Enfermedades y salud & 9 & 9 & 19 \\
\hline Drogas y alcohol & 4 & 6 & 8 & Tecnología & 7 & 9 & 20 \\
\hline Educación & 9 & 10 & 20 & \begin{tabular}{|l} 
Televisión sobre \\
televisión
\end{tabular} & 7 & 14 & 21 \\
\hline Emigración e inmigración & 3 & 7 & 10 & Terrorismo & 4 & 6 & 13 \\
\hline Medio ambiente & 5 & 7 & 21 & Turismo y vacaciones & 5 & 5 & 17 \\
\hline Integración europea & 5 & 10 & 13 & Transportes & 5 & 4 & 20 \\
\hline Familia & 4 & 11 & 26 & Guerras y conflictos & 2 & 10 & 19 \\
\hline Moda & 7 & 10 & 18 & Estado del bienestar & 2 & 10 & 15 \\
\hline Alimentación & 4 & 5 & 29 & Trabajo & 3 & 3 & 22 \\
\hline Revolución de género & 2 & 3 & 28 & Cultura juvenil & 7 & 6 & 12 \\
\hline
\end{tabular}

Tabla 1. Selección temática de TVC

\section{"Las principales dificultades se han localizado en dos aspectos muy concretos: en la titularidad de los derechos de propiedad intelectual y en la selección temática"}

Más allá de las lógicas dificultades técnicas que surgen en todo proyecto de esta envergadura, las principales dificultades se han localizado en dos aspectos muy concretos: en la titularidad de los derechos de propiedad intelectual y en la selección temática. Sobre el primer aspecto, se desconocía a quién pertenecían los derechos de determinadas obras dados los años transcurridos desde que se crearon. En otros casos la progresiva externalización de la producción realizada por los operadores televisivos en los últimos años ha generado 
discusiones sobre la propiedad de estos derechos y su explotación. Sobre la selección temática, las propias diferencias sociales y culturales de los países respectivos han dificultado la localización de materias afines o con posibilidades de comparación.

A pesar de estos obstáculos, $\mathrm{Vi}$ deoActive se ha puesto en marcha y ya constituye un activo electrónico muy relevante para docentes e investigadores del campo de la comunicación. Además, desde el consorcio se persigue la continuación del proyecto ampliándolo cuantitativa y cualitativamente, y se plantea la posibilidad de abrirlo a otros operadores y organismos interesados.

\section{Bibliografía}

Agirreazaldegi-Berriozabal, Teresa. "Claves y retos de la documentación digital en televisión". El profesional de la información, 2007, septiembre-octubre, v. 16, n. 5, pp. 433-442.

Besser, Howard. "Collaboration for electronic preservation". Library trends, 2007, verano, v. 56, n. 1, pp. 216-229.

Comisión de las Comunidades Europeas. Comunicación de la Comisión al Parlamento Europeo al Consejo, al Comité Económico y Social Europeo y al Comité de las Regiones. 12010 Bibliotecas Digitales, COM(2005) 465 final. Bruselas: Comisión Europea, 2005.

http://eur-lex.europa.eu/LexUriServ/LexUriServ. do? uri=COM:2005:0465:FIN:ES:PDF

De-Moragas, Miquel; Prado, Emili. La televisió pública a l'era digital. Barcelona: Pòrtic, 2000, ISBN 84-7306-617-0.

Gómez-Alonso, Rafael. "Investigar la historia de la televisión en España: algunos problemas documentales y metodológicos". Área abierta, 2004, enero, n. 7.

Hecht, Alexander; O'Dwyer, Andrew; Oomen, Johan; Scharinger, Florian. "Birth: building an interactive research and delivery network for television heritage”. En: Proceedings of Ichim digital culture and heritage, 2004

http://www.ichim.org/ichim04/contenu/PDF/ 2280_Oomen.pdf

Lloyd, Annemare. "Guarding against collective amnesia? Making significance problematic: an exploration of issues". Library trends, 2007 , verano, v. 56, n. 1, pp. 53-65.

Prado, Emili; Fernández-Quijada, David "The role of public service broadcasters in the era of convergence. A case study of Televisió de Catalunya". Communications \& strategies, 2006, n. 62, pp. 49-69.

http://www.idate.fr/fic/revue_telech/148/CS62_ PRADO_FERNANDEZ.pdf
Teruggi, Daniel. "Can we save our audio-visual heritage?". Ariadne, 2004, abril, n. 39

http://www.ariadne.ac.uk/issue39/teruggi/

Wright, Richard. "Digital preservation of audio, video and film". Vine: The journal of information and knowledge management systems, 2004, v. 34, n. 2.

David Fernández-Quijada, Departament de Comunicació Audiovisual i de Publicitat, Universitat Au- tònoma de Barcelona. Edifici I, Facultat de Ciències de la Comunicació. Campus de Bellaterra. 08193 Bellaterra (Barcelona).

david.fernandez@uab.es

Montse Fortino, Departament de Documentació, Televisió de Catalunya. c/ de la TV3, s/n.08970 Sant Joan Despí (Barcelona). mfortino.b@tv3.cat

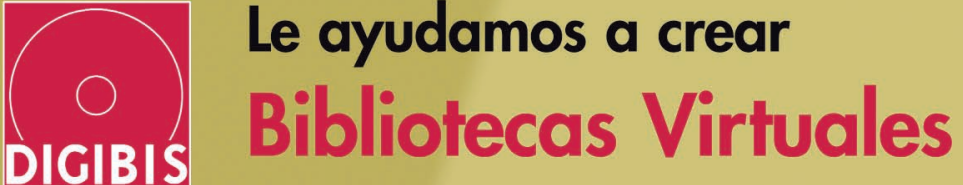

desde la digitalización de materiales bibliográficos hasta la asignación de metadaios y su implementación en la red, conforme a la normativa internacional

\section{Productos para crear Bibliotecas Digitales y Virtuales}

DICIBIB 5.0

Solución avanzada para la creación de Bibliotecas Digitales y la Gestión Bibliotecaria Multilingüe DICIARCH 1.6 Sistema digital de descripción y gestión archivística

Digifalización avonzacle Con asignación dinámica de metadatos

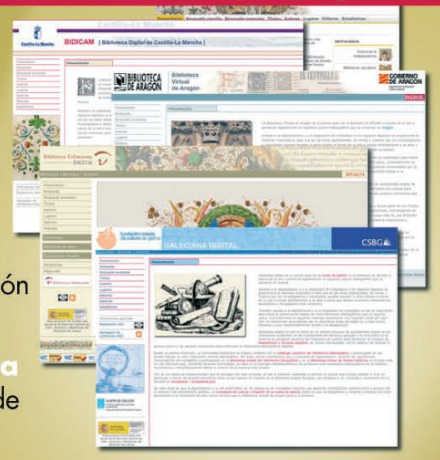

OAsls-PMH 2.0 Sistema integrado de recolección de diversos esquemas de metadatos:

DCMI sin cualificar MARC 21

EAD

mod_OAl

Implementación de las directrices DRIVER
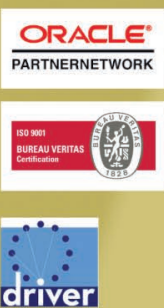

Recolección en la Web para Entidades e Instituciones de Memoria en OAl-PMH y Dublin Core e intercambio de metadatos en METS (diferentes Profiles)

- Consultoría y mappings a DCMI para implementación en repositorios OAl v2.0

Tecnologías abiertas para la creación, recuperación y recolección de metadatos (MARCXML, DCMI y RDF)

Repositorios Institucionales para Preservación Digital a largo plazo mediante PREMIS y OAIS ISO 14721

- Reconocimiento Óptico de Caracteres OCR Y generación dinámica de METS/Alio

- Servidor adicional de SRU Search/Retrieval via URL SRU

- Agregadores de contenido RSS A

Validación en el Data Providers de la Open Archives Intiative. Genera un Sitemap para Google.

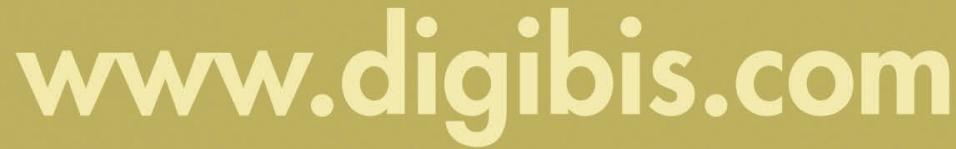

